\title{
Coocorrência de violência física e psicológica entre adolescentes namorados do recife, Brasil: prevalência e fatores associados
}

\author{
Co-occurrence of physical and psychological violence \\ among dating adolescents in Recife, Brazil: \\ prevalence and associated factors
}

Alice Kelly Barreira ${ }^{1}$

Maria Luiza Carvalho de Lima ${ }^{1}$

Joviana Quintes Avanci ${ }^{2}$

${ }^{1}$ Núcleo de Estudos em Saúde Coletiva, Centro de pesquisas Aggeu Magalhães, Fiocruz. Rua Prof. Moraes Rego $s / n$, Cidade

Universitária. 50670-420 Recife PE

${ }^{2}$ Departamento de Epidemiologia e Métodos Quantitativos em Saúde, Escola Nacional de Saúde Pública, Fundação Oswaldo Cruz.

\begin{abstract}
The scope of this study was to assess the prevalence of physical and psychological violence among dating adolescents from Recife, Brazil, to identify associated factors and the co-occurrence of both types of violence. 302 adolescents aged between 15 and 19 years in public and private schools who were in a relationship in the last year filled out the questionnaire. Univariate and multivariate logistic regression statistical analysis were performed and the sample weight and the complex sample design were entered. The prevalence was $19.9 \%$ of physical violence $82.8 \%$ of psychological violence and $18.9 \%$ for the co-occurrence of both. Adolescents who have experienced violence in the community and who were in dating relationships of more than one year duration were more likely to perpetrate psychological violence. For the co-occurrence of physical and psychological violence, the variables that increased the chance of perpetration were suffering physical violence from the father, violence among siblings, physical violence and the perpetration of verbal violence in previous relationships. The conclusion reached is that psychological violence and the co-occurrence of physical and psychological violence have a distinct dynamics from physical violence in dating relationships.
\end{abstract}

Key words Adolescents, Violence, Risk factors
Resumo Os objetivos foram estimar a prevalência de perpetração de violência física e psicológica entre adolescentes namorados de Recife, identificar fatores associados e a coocorrência de ambos os tipos de violência. Participaram do estudo 302 adolescentes de escolas públicas e particulares, com idade entre 15 e 19 anos, que tiveram algum relacionamento amoroso no último ano e esses preencheram o questionário. Foram realizadas análise univariada e regressão logística e todas as análises estatísticas incorporaram o peso amostral e o desenho da amostra complexa. A prevalencia de violência física foi de 19,9\%, de 82,8\% para violência psicológica e de 18,9\% para a coocorrência de violência física e psicológica. Os adolescentes que vivenciaram violência na comunidade e em relacionamentos de mais de um ano de duração apresentaram maiores chances de perpetrar violência psicológica. Enquanto, sofrer violência física do pai, entre irmãos e em namoros anteriores, além de ter perpetrado violência verbal em relacionamentos anteriores, foram variáveis que aumentaram a chance de perpetração de violência física e psicológica no namoro. Conclui-se que a violência psicológica e a coocorrência de violência física e psicológica possuem uma dinâmica distinta da violência física no namoro.

Palavras-chave Adolescentes, Violência, Fatores de risco 


\section{Introdução}

O estudo da violência nas relações afetivo-sexuais de jovens e adolescentes (namoro, ficar) deriva das pesquisas sobre a violência contra a mulher nas relações entre parceiros íntimos na vida adulta, que tiveram início a partir do esforço do movimento feminista em denunciar a violência doméstica na década de 1970. Atualmente, sabese que a violência nas relações afetivo-sexuais de jovens e adolescentes, além de representar um potencial precursor da violência entre parceiros íntimos na fase adulta ${ }^{1}$, tem especificidades próprias da faixa etária e é tão grave quanto essa, em termos de prevalência, lesões e danos psicológicos à vítima; e, portanto, deve ser estudada independentemente ${ }^{2}$.

Usualmente denominada violência no namoro (dating violence, courtship violence, violence amoureuse), a violência nas relações íntimas de adolescentes inclui agressão física, abuso psicológico e sexual. Jovens e adolescentes de ambos os sexos podem ser vítimas e/ou perpetradores da violência, mas, geralmente têm dificuldade em reconhecer a violência como tal $^{3}$ e raramente procuram ajuda ${ }^{4,5}$.

As pesquisas que abordam as relações de namoro de adolescentes apontam sempre altas prevalências de violência, mas que apresentam ampla variação, em torno de: $10 \%^{6}, 15 \%{ }^{7,8}, 20 \%{ }^{9}$, $40 \%^{10,11}, 80 \%^{12,13}$. A disparidade nos dados de prevalência é frequentemente atribuída à utilização de diferentes metodologias e à ausência de um padrão conceitual ${ }^{14}$. Em pesquisa realizada em jovens universitários de 32 nações, incluindo o Brasil, $17 \%$ a $49 \%$ deles relataram ter agredido fisicamente o parceiro no último ano, com média de 29\%. A prevalência de agressões físicas mais severas, como esmurrar, estrangular e agredir com armas foi em média $10 \%{ }^{15}$.

Na maioria dos estudos o foco tem sido principalmente para a violência física, enquanto há uma atenção muito limitada à agressão psicológica ou sexual ${ }^{16}$. Mesmo quando variadas formas de violência são avaliadas, essas têm sido abordadas isoladamente uma das outras, ou então são estudadas como uma questão única. Dessa forma, pouca visibilidade é dada ao fenômeno denominado de polivitimização ou coocorrência da violência, entendido pela existência de vitimização ou de perpetração, respectivamente, simultânea de mais de um tipo de violência física, psicológica ou sexual ${ }^{17,18}$.

Os poucos estudos que abordaram violência física e psicológica no namoro sugeriram sua associação ${ }^{19,20}$, onde a violência psicológica pode ser um indicador de violência física simultânea ou longitudinalmente ${ }^{20}$. Sears et al. ${ }^{18}$ avaliaram a coocorrência de violência física, psicológica e sexual, e observaram que $19 \%$ dos garotos e $26 \%$ das garotas relataram ter feito uso de pelo menos duas formas de violência.

O conhecimento dos fatores de risco para a violência no namoro é primordial para a interrupção do ciclo de violência por meio de intervenções e tratamentos efetivos. Infelizmente, a dificuldade de encontrar consenso na literatura sobre violência no namoro também é válida para o estudo dos fatores de risco. Os trabalhos compartilham poucas variáveis comuns na investigação sobre fatores de risco ou proteção ${ }^{21}$. Além disso, avaliam poucos fatores de cada vez e, geralmente, na ausência de modelos teóricos explicativos. Para Foshee et al..$^{22}$, muitas das associações observadas podem ter sido confundidas por outras variáveis associadas tanto com o fator estudado como com a violência no namoro e que não foram incluídas nas pesquisas.

Embora exista uma vasta literatura internacional sobre a violência no namoro, no Brasil apenas recentemente o tema tem despertado o interesse da comunidade científica ${ }^{23,24}$. A pouquíssima produção nacional sobre o assunto e a inadequação em utilizar dados de outros países e de diferentes culturas alertam para a necessidade de estudos que investiguem a prevalência, os fatores associados e as especificidades da violência no namoro em adolescentes e jovens brasileiros.

O presente estudo teve como objetivos estimar a prevalência de violência física e psicológica entre namorados adolescentes de Recife, assim como identificar seus fatores associados e a coocorrência de ambos os tipos de violência investigados.

\section{Método}

Esta pesquisa é um recorte de um projeto de delineamento transversal, realizado em 10 capitais do Brasil no ano de 2008, do qual neste trabalho somente os dados referentes à cidade do Recife serão apresentados ${ }^{24}$. O estudo foi aprovado pelo Comitê de Ética em Pesquisa da Escola Nacional de Saúde Pública Sérgio Arouca. Autorização para a pesquisa também foi dada por escrito pela Secretaria Estadual de Educação de Pernambuco. A direção das escolas envolvidas e os alunos que participaram da investigação assinaram um termo de Consentimento Livre e Esclarecido, conforme preconizado na Resolução 196/96 do Con- 
selho Nacional de Saúde, que normaliza as pesquisas com seres humanos ${ }^{25}$.

Foi alvo desse estudo a população de adolescentes matriculados no segundo ano de ensino médio de escolas públicas (estaduais) e particulares, com idade entre 15 e 19 anos, da cidade do Recife.

O recorte de alunos no segundo ano foi tomado devido à maior facilidade desse grupo em responder a temas mais delicados como o da sexualidade, e por não estarem ainda no último ano, etapa em que as escolas têm mais dificuldade em permitir a liberação de período para pesquisa. Só participaram alunos do curso diurno (7 às 19 horas), pois os alunos do curso noturno são mais velhos e apresentam características muito distintas.

O plano amostral definiu dois estratos, em função da natureza da instituição: pública e particular, para garantir a representatividade segundo estratos socioeconômicos. Adotou-se a amostragem pelas proporções de cada estrato, com uma amostra aleatória simples. A seleção se deu em múltiplos estágios. Na $1^{\text {a }}$ etapa foram selecionadas as escolas com probabilidade de seleção proporcional à quantidade de alunos do $2^{\circ}$ ano; e na $2^{\text {a }}$ etapa uma turma foi selecionada aleatoriamente, dentro da escola, para a aplicação do questionário com todos os alunos. O plano amostral foi assim delineado com o objetivo de encontrar menor tamanho amostral com maior precisão e poder de inferência para a população das cidades envolvidas ${ }^{26}$.

O tamanho amostral estimado para a cidade de Recife foi de 320 alunos da $2^{\text {a }}$ série do ensino médio distribuído igualmente pelos estratos de natureza da escola, ao nível de 99\% de confiança, erro relativo de $5 \%$ e prevalência de $70 \%$ para violência (prevalência encontrada na amostra de Manaus, primeira cidade a ser pesquisada e que serviu de referência para todo o estudo). Os dados utilizados para o cálculo se referem ao número de matrículas no $2^{\circ}$ ano do ensino médio do curso diurno no ano de 2006 fornecido pela Secretaria de Educação e Cultura de Pernambuco.

Não houve nenhuma recusa de aluno em participar da pesquisa. A amostra obtida foi de 355 adolescentes, sendo que 53 foram excluídos de acordo com os critérios: idade não informada e nunca ter "ficado" ou namorado. De forma que a amostra final para análise foi de 302 adolescentes, sendo 133 da rede de ensino público e 169 do ensino privado.

O instrumento, elaborado pela equipe de pesquisadores do Centro Latino-Americano de Es- tudos de Violência e Saúde Jorge Careli (Claves), consistiu de um questionário fechado de autopreenchimento, que foi pré-testado.

Utilizou-se o Conflict in Adolescent Dating Relationship Inventory - Cadri ${ }^{8}$ para avaliar a violência nos relacionamentos afetivos dos adolescentes (independentemente da idade do parceiro/a), por ser uma escala criada especificamente para o universo de adolescentes e adaptada para língua portuguesa ${ }^{24}$. É uma escala com 70 itens, dos quais 25 aferem violência sofrida, 25 referem-se à violência perpetrada e 20 são itens que distraem o jovem da ênfase no tema da violência, não fazendo parte da análise da escala. A Cadri afere cinco formas de violência presentes no relacionamento amoroso entre adolescentes: a) física; b) sexual; c) psicológica, discriminada em três subtipos: ameaças, verbal/emocional e relacional. Neste trabalho, os itens da escala que aferem violência sexual não foram utilizados.

$\mathrm{Na}$ escala Cadri adaptada para a língua portuguesa ${ }^{24}$, os índices de correlação intraclasse (ICC) encontrados para a violência perpetrada indicam estabilidade teste-reteste satisfatória, segundo critério descrito por Szklo e Nieto ${ }^{27}$, com valores acima de 0.4: 0,497 (IC 95\% 0,422-0,566) para violência física, 0,459 (IC 95\% 0,381-0,531) para ameaças, 0,497 (IC 95\% 0,421-0,566) para violência relacional e 0,701 (IC 95\% 0,637-0,754) para violência verbal. A análise do Alpha de Cronbach na amostra revelou boa consistência interna para a maioria das violências, com exceção da violência relacional que apresenta uma consistência interna mais reduzida. Vale destacar que apenas 3 itens compõem a violência relacional, o que pode influir nos resultados obtidos: 0,819 para violência física perpetrada; 0,691 para ameaça perpetrada; 0,539 para violência relacional perpetrada; 0,844 para violência verbal emocional perpetrada.

As variáveis perpetração de violência psicológica e perpetração de violência física e psicológica concomitantemente, chamada de coocorrência foram consideradas dependentes no estudo. A presença de violência foi considerada quando existiram um ou mais itens afirmativos de perpetração de violência.

Como variáveis independentes, outras questões foram aferidas para contextualizar as relações de violência nas relações íntimas de adolescentes. As questões foram agrupadas segundo as dimensões do modelo bioecológico de Bronfrebrenner $^{28}$, listadas a seguir.

a) Fatores de processo: violência na escola e violência na comunidade ${ }^{29}$; ter sofrido violência 
verbal e física da mãe, ter sofrido violência verbal e física do pai (Conflict Tactics Scale ${ }^{30}$ ); já ter brigado com irmãos a ponto de se machucarem; ter sofrido violência verbal, física e sexual em relacionamentos anteriores; monitoramento dos pais; e importância atribuída ao namorado(a).

b) Fatores pessoais: sexo; cor da pele; religião; uso de álcool; uso de drogas; autoestima (escala de Rosenberg, 1989 adaptada por Avanci et al. ${ }^{31}$ ); desempenho escolar; aceitação da violência feminina no namoro; aceitação da violência masculina no namoro.

c) Fatores de contexto: arranjo familiar; extrato socioeconômico (escala $\mathrm{ABEP}^{32}$ ); escolaridade da mãe; testemunho de violência na comunidade; testemunho de violência psicológica entre os pais; testemunho de violência física entre os pais.

d) Fatores relacionados ao tempo: duração do relacionamento; idade que começou a ficar, namorar e transar; ter praticado violência verbal nos relacionamentos anteriores; ter praticado violência física nos relacionamentos anteriores.

Todas as análises estatísticas incorporaram: $\mathrm{o}$ peso amostral, a fim de que fossem corrigidas as estimativas pontuais (como, por exemplo, os percentuais) e o desenho amostral, visando corrigir as estimativas de variância. Essa opção deveu-se à menor estimativa de variância, característica dos desenhos amostrais conglomerados, em comparação com os testes estatísticos normalmente utilizados em amostra aleatória simples. As análises foram feitas com o pacote de análise para amostra complexa do software SPSS 17.0.

Os dados foram analisados através da estatística descritiva (frequência absoluta e relativa de violência física, psicológica e coocorrência, segundo o sexo). Para identificar a associação entre as variáveis independentes e violência psicológica e coocorrência, foi realizada a análise univariada. Foi utilizado o teste do qui-quadrado, quando indicado, com nível de significância de $20 \%$ para selecionar as variáveis para o modelo multivariado de regressão logística. A seleção de variáveis dentro da análise multivariada foi realizada utilizando o algorítimo Forward Stepwise, com probabilidade de entrada no modelo de $5 \%$.

\section{Resultados}

Do total de 302 adolescentes que participaram do estudo, $56,3 \%$ eram do sexo feminino e a maioria se considerava de cor branca $(41,7 \%)$ ou parda $(37,3 \%)$. A maior parte da amostra foi composta de estudantes da rede de ensino privado $(56,0 \%)$ e representantes dos extratos sociais A e B $(54,1 \%)$. Com relação à escolaridade de pai e mãe, observou-se um percentual mínimo de analfabetismo ( $0,7 \%$ e $2,4 \%$, respectivamente), com $45,6 \%$ dos pais e $39,7 \%$ das mães tendo cursado o ensino médio. A maioria dos adolescentes $(74,0 \%)$ afirmou praticar alguma religião.

Sessenta adolescentes $(19,9 \%)$ relataram perpetrar violência física contra os seus parceiros, sendo 37 meninas $(21,8 \%)$ e 23 meninos $(17,4 \%)$. Para violência psicológica, a prevalência de perpetração foi de $82,8 \%$, sendo $80,6 \%$ no sexo feminino e $85,6 \%$ no sexo masculino. A prevalência de coocorrência de violência física e psicológica foi de $18,9 \%$, com $20,6 \%$ para o sexo feminino e $16,7 \%$ para o sexo masculino. Para todas as prevalências, a diferença entre os sexos não foi significante, com $\mathrm{p}>0,05$.

As Tabelas 1 a 4 apresentam os resultados da associação entre violência (psicológica e coocorrência de física e psicológica) com variáveis independentes na análise univariada, segundo as dimensões do modelo bioecológico. As variáveis que apresentaram associação com violência psicológica, com $p<0,20$, foram: violência na escola, violência na comunidade, ter sofrido violência verbal da mãe, ter sofrido violência verbal do pai, ter sofrido violência física do pai, ter sofrido violência verbal em relacionamentos anteriores, importância atribuída ao namorado(a), uso de álcool, aceitação da violência masculina no namoro, escolaridade da mãe, testemunho de violência na comunidade, duração do relacionamento, idade que começou a ficar, e ter praticado violência verbal nos relacionamentos anteriores.

Para identificar a associação entre coocorrência e as variáveis independentes, foram selecionadas $(p<0,20)$ : violência na escola, violência na comunidade, ter sofrido violência verbal da mãe, ter sofrido violência física da mãe, ter sofrido violência verbal do pai, ter sofrido violência física do pai, violência entre irmãos, ter sofrido violência verbal em relacionamento anterior, ter sofrido violência física em relacionamento anterior, monitoramento dos pais (Tabela 1), aceitação da violência feminina no namoro, aceitação da violência masculina no namoro (Tabela 2), arranjo familiar, extrato socioeconômico, escolaridade da mãe, testemunho de violência física entre os pais (Tabela 3), ter perpetrado violência verbal em relacionamento anterior e ter perpetrado violência física em relacionamento anterior (Tabela 4).

No modelo final, após o ajuste com todas as variáveis selecionadas na etapa anterior, obser- 
Tabela 1. Análise univariada da associação entre violência e os fatores de processo, em adolescentes namorados. Recife, 2008.

\begin{tabular}{|c|c|c|c|c|c|c|c|c|}
\hline \multirow{2}{*}{ Fatores de processo } & \multicolumn{4}{|c|}{ Violência psicológica } & \multicolumn{4}{|c|}{ Violência física e psicológica } \\
\hline & $\%$ & OR & $\mathrm{IC}_{95 \%}(\mathrm{OR})$ & p-valor & $\%$ & OR & $\mathrm{IC}_{95 \%}(\mathrm{OR})$ & p-valor \\
\hline \multicolumn{9}{|l|}{ Sofre violência na escola } \\
\hline Não & 46,2 & 1,00 & - & - & 36,2 & 1,00 & - & - \\
\hline Sim & 53,8 & 2,02 & $1,26-3,25$ & 0,008 & 63,8 & 1,95 & $1,09-3,52$ & 0,028 \\
\hline \multicolumn{9}{|l|}{ Sofre violência na comunidade } \\
\hline Não & 40,8 & 1,00 & - & - & 25,9 & 1,00 & - & - \\
\hline Sim & 59,2 & 3,84 & $1,72-8,59$ & 0,003 & 74,1 & 3,01 & $1,15-7,88$ & 0,025 \\
\hline \multicolumn{9}{|l|}{ Sofre violência verbal da mãe } \\
\hline Não & 17,1 & 1,00 & - & - & 10,8 & 1,00 & - & - \\
\hline Sim & 82,9 & 1,87 & $0,80-4,40$ & 0,128 & 89,2 & 2,19 & $0,92-5,24$ & 0,067 \\
\hline \multicolumn{9}{|l|}{ Sofre violência física da mãe } \\
\hline Não & 45,7 & 1,00 & - & - & 24,9 & 1,00 & - & - \\
\hline Sim & 54,3 & 0,97 & $0,47-2,04$ & 0,939 & 75,1 & 3,08 & $1,73-5,46$ & 0,001 \\
\hline \multicolumn{9}{|l|}{ Sofre violência verbal do pai } \\
\hline Não & 28,3 & 1,00 & - & - & 17,1 & 1,00 & - & - \\
\hline Sim & 71,7 & 1,63 & $0,86-3,07$ & 0,115 & 82,9 & 2,40 & $1,09-5,30$ & 0,030 \\
\hline \multicolumn{9}{|l|}{ Sofre violência física do pai } \\
\hline Não & 65,9 & 1,00 & - & - & 38,5 & 1,00 & - & - \\
\hline $\operatorname{Sim}$ & 34,1 & 2,38 & $1,03-5,50$ & 0,041 & 61,5 & 4,99 & $2,47-10,08$ & 0,000 \\
\hline \multicolumn{9}{|l|}{ Violência física entre irmãos } \\
\hline Não & 54,1 & 1,00 & - & - & 29,4 & 1,00 & - & - \\
\hline $\operatorname{Sim}$ & 45,9 & 0,72 & $0,39-1,34$ & 0,264 & 70,6 & 3,29 & $1,35-8,06$ & 0,012 \\
\hline \multicolumn{9}{|l|}{$\begin{array}{l}\text { Sofreu violência verbal em } \\
\text { relacionamentos anteriores }\end{array}$} \\
\hline Não & 71,7 & 1,00 & - & - & 55,4 & 1,00 & - & - \\
\hline $\operatorname{Sim}$ & 28,3 & 1,63 & $1,01-2,62$ & 0,045 & 44,6 & 2,77 & $1,60-4,78$ & 0,002 \\
\hline \multicolumn{9}{|l|}{$\begin{array}{l}\text { Sofreu violência física em } \\
\text { relacionamentos anteriores }\end{array}$} \\
\hline Não & 98,1 & 1,00 & - & - & 95,4 & 1,00 & - & - \\
\hline $\operatorname{Sim}$ & 1,9 & 0,96 & $0,10-9,07$ & 0,965 & 4,6 & 3,61 & $0,62-20,86$ & 0,112 \\
\hline \multicolumn{9}{|l|}{$\begin{array}{l}\text { Sofreu violência sexual em } \\
\text { relacionamentos anteriores }\end{array}$} \\
\hline Não & 97,6 & 1,00 & - & - & 98,1 & 1,00 & - & - \\
\hline Sim & 2,4 & 1,03 & $0,08-13,07$ & 0,981 & 1,9 & 0,75 & $0,09-6,00$ & 0,761 \\
\hline \multicolumn{9}{|l|}{ Monitoramento dos pais } \\
\hline Monitora & 85,5 & 1,00 & - & - & 82,9 & 1,00 & - & - \\
\hline Não monitora & 14,5 & 2,30 & $0,40-13,09$ & 0,295 & 17,1 & 1,49 & $0,88-2,50$ & 0,117 \\
\hline \multicolumn{9}{|c|}{ Importância atribuída ao parceiro } \\
\hline Importante/Muito & 81,5 & 1,00 & - & - & 78,7 & 1,00 & - & - \\
\hline Pouco & 18,5 & 0,53 & $0,31-0,91$ & 0,026 & 21,3 & 1,06 & $0,59-1,92$ & 0,814 \\
\hline
\end{tabular}

vou-se que adolescentes namorados que vivenciam violência na comunidade apresentaram 3,99 mais chances de perpetrar violência psicológica, e que em relacionamentos com mais de um ano de duração as chances dessa violência são maiores quando comparadas com relacionamentos com menos de um mês de duração (Tabela 5). 


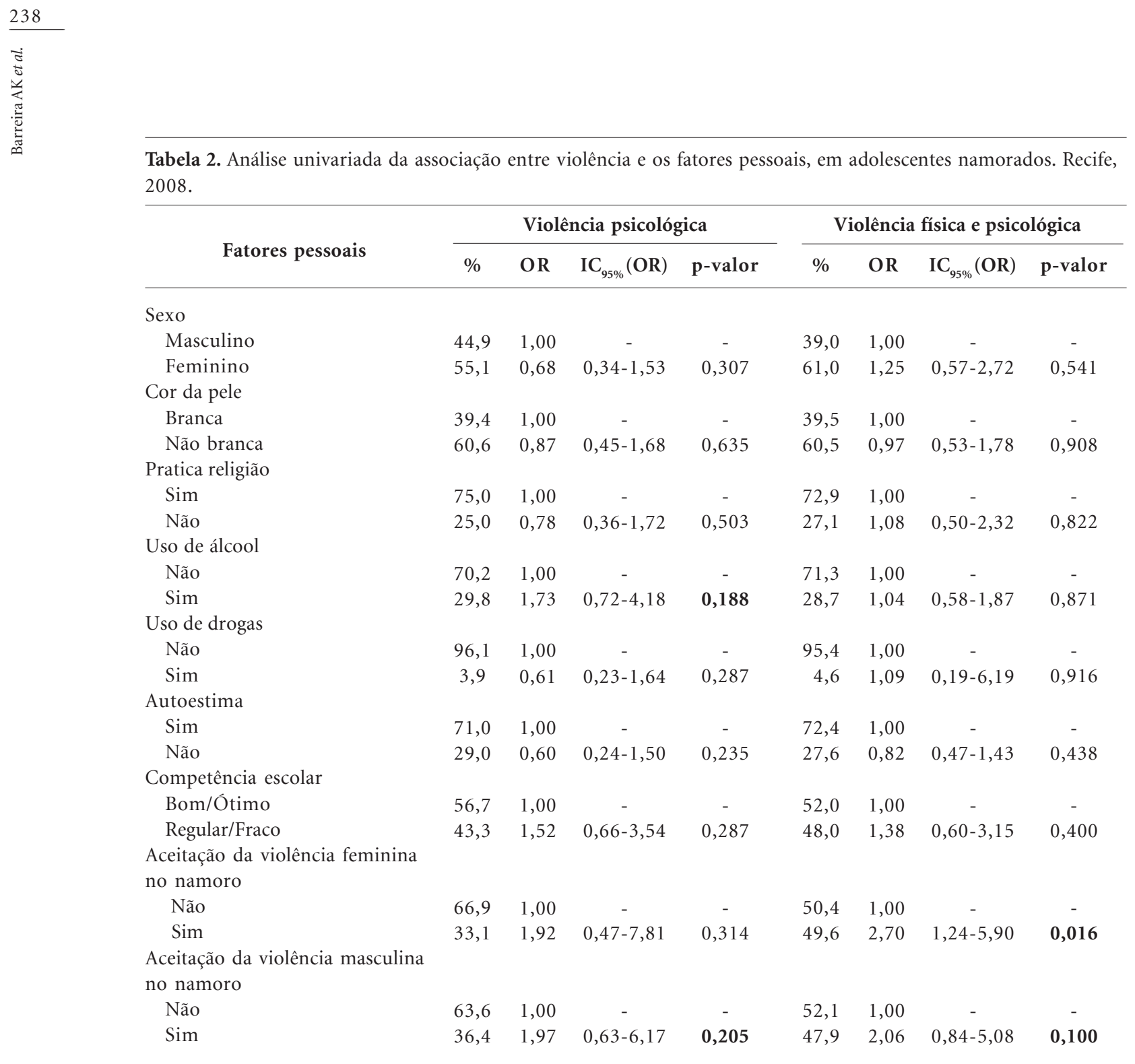

\section{Discussão}

No presente estudo, observou-se que 19,9\% dos adolescentes que tiveram relacionamentos amorosos no último ano perpetraram algum ato de violência física e 82,8\% de violência psicológica. Esse achado corrobora os dados encontrados na literatura, na qual a violência psicológica tende a ser muito mais prevalente do que a violência física entre namorados adolescentes, podendo atingir prevalências de $50 \%{ }^{8}$, a $80 \%{ }^{10,11,13}$, enquanto a violência física apresenta prevalência em torno de $10 \%$ a $20 \%{ }^{15}$.

Um aspecto fundamental, que perpassa o problema de definição da violência e sua mensuração, refere-se ao tipo ou tipos de violência avaliados em uma dada pesquisa. Os estudos sobre violência no namoro são mais frequentes para agressão física e, portanto, adotam o conceito mais estreito de violência. As consequências de excluir os outros tipos de violência, como psicológica e sexual, é que os dados disponíveis na literatura podem estar subestimando consideravelmente a extensão do fenômeno ${ }^{16}$. Recentemente, mais pesquisas têm incluído outros tipos de violência além da física e, portanto, apresentam taxas mais elevadas de violência no namoro entre adolescentes ${ }^{12,13,33,34}$.

$\mathrm{Na}$ amostra estudada, dentre os 60 adolescentes que afirmaram perpetrar violência física no namoro, 57 também perpetram a violência psicológica, o que representa uma prevalência de $18,9 \%$ de coocorrência e evidencia que a violência física raramente ocorre na ausência da psicológica. Outros estudos também concluíram que os atos de agressão física estão quase sempre acom- 
Tabela 3. Análise univariada da associação entre violência e os fatores de contexto, em adolescentes namorados. Recife, 2008.

\begin{tabular}{|c|c|c|c|c|c|c|c|c|}
\hline \multirow{2}{*}{ Fatores de contexto } & \multicolumn{4}{|c|}{ Violência psicológica } & \multicolumn{4}{|c|}{ Violência física e psicológica } \\
\hline & $\%$ & OR & $\mathrm{IC}_{95 \%}(\mathrm{OR})$ & p-valor & $\%$ & OR & $\mathrm{IC}_{95 \%}(\mathrm{OR})$ & p-valor \\
\hline \multicolumn{9}{|l|}{ Arranjo familiar } \\
\hline Pai e mãe & 58,7 & 1,00 & - & - & 68,7 & 1,00 & - & - \\
\hline Outros & 41,3 & 0,95 & $0,65-1,40$ & 0,778 & 31,3 & 0,58 & $0,35-0,97$ & 0,038 \\
\hline \multicolumn{9}{|l|}{ Extrato socioeconômico } \\
\hline $\mathrm{A} 1, \mathrm{~A} 2, \mathrm{~B} 1, \mathrm{~B} 2$ & 50,3 & 1,00 & - & - & 33,9 & 1,00 & - & - \\
\hline $\mathrm{C}, \mathrm{D}, \mathrm{E}$ & 49,7 & 0,92 & $0,38-2,22$ & 0,828 & 66,1 & 2,26 & $1,02-4,98$ & 0,043 \\
\hline \multicolumn{9}{|l|}{ Escolaridade da mãe } \\
\hline Ensino superior & 24,3 & 1,00 & - & - & 16,3 & 1,00 & - & - \\
\hline E. médio completo & 34,5 & 1,23 & $0,34-4,48$ & 0,070 & 34,5 & 1,67 & $0,82-3,40$ & 0,087 \\
\hline E. fund. completo & 22,9 & 1,96 & $0,96-3,98$ & 0,070 & 21,8 & 1,68 & $0,67-4,24$ & 0,087 \\
\hline E. fund. incompleto & 15,5 & 0,48 & $0,15-1,47$ & 0,070 & 18,7 & 1,63 & $0,46-5,84$ & 0,087 \\
\hline Não sabe ler/escrever & 2,8 & 1,36 & $0,08-24,89$ & 0,070 & 8,6 & 10,07 & $1,59-63,69$ & 0,087 \\
\hline \multicolumn{9}{|l|}{$\begin{array}{l}\text { Testemunho de violência } \\
\text { na comunidade }\end{array}$} \\
\hline Não & 11,1 & 1,00 & - & - & 9,5 & 1,00 & - & - \\
\hline Sim & 88,9 & 1,73 & $0,77-3,89$ & 0,160 & 90,5 & 1,42 & $0,44-4,60$ & 0,518 \\
\hline \multicolumn{9}{|l|}{$\begin{array}{l}\text { Testemunho de violência } \\
\text { psicológica entre os pais }\end{array}$} \\
\hline Não & 78,2 & 1,00 & - & - & 71,3 & 1,00 & - & - \\
\hline Sim & 21,8 & 1,379 & $0,50-3,79$ & 0,489 & 28,7 & 1,70 & $0,56-5,15$ & 0,301 \\
\hline \multicolumn{9}{|l|}{$\begin{array}{l}\text { Testemunho de violência } \\
\text { física entre os pais }\end{array}$} \\
\hline Não & 90,9 & 1,00 & - & - & 84,2 & 1,00 & - & - \\
\hline Sim & 9,1 & 1,508 & $0,46-4,95$ & 0,452 & 15,8 & 2,50 & $1,08-5,78$ & 0,032 \\
\hline
\end{tabular}

panhados da agressão psicológica concomitan$\mathrm{te}^{11,20}$, exemplificada por abuso verbal, relacional e ameaças.

Pesquisa realizada nos Estados Unidos com 633 adolescentes escolares observou que $6 \%$ dos meninos e $23 \%$ das meninas afirmaram ter usado violência física e psicológica no namoro ao mesmo tempo ${ }^{18}$. A maior prevalência de perpetração, seja de violência física e/ou psicológica, para as adolescentes do sexo feminino é um achado comum na literatura sobre violência no namoro, entretanto, nos resultados aqui apresentados essa diferença não foi significante, provavelmente devido ao limitado tamanho da amostra.

Existem poucos trabalhos na literatura que estudaram os fatores associados à violência psicológica, e menos ainda à coocorrência de violência física e psicológica. Por isso, foi investigada a literatura em geral sobre violência no namoro, principalmente sobre violência física, para identificar os possíveis fatores de risco a serem incluídos na análise. Também por esse motivo, a discussão a seguir sobre os fatores associados não possui dados específicos sobre violência psicológica ou sobre coocorrência para serem confrontados.

Por outro lado, os resultados da presente pesquisa sugerem que a violência psicológica e a coocorrência de violência física e psicológica possuem uma dinâmica distinta da violência física no namoro, uma vez que fatores de risco frequentemente destacados nos estudos sobre agressão física, como uso de álcool e drogas ${ }^{22,33,35-37}$, autoestima ${ }^{6,36}$, aceitação da violência ${ }^{36-39}$ e testemunho de violência entre os pais ${ }^{9,36,38,40}$, não mostraram associação com os tipos de violência aqui estudados.

Foi observado que adolescentes que vivenciam violência na comunidade apresentaram quase quatro vezes mais chances de perpetrar violência psicológica no namoro. Em outros estudos, a exposição à violência na comunidade também tem sido associada à perpetração de violência no namoro entre adolescentes de ambos os sexos $^{36,41}$. Para O'Keefe ${ }^{14}$ tal associação ocorre pelo aumento da aceitação da violência em indivíduos expostos à violência na comunidade. Garbarino $^{42}$ também alerta para a influência de ambien- 
Tabela 4. Análise univariada da associação entre violência e os fatores relacionados ao tempo, em adolescentes namorados. Recife, 2008.

\begin{tabular}{|c|c|c|c|c|c|c|c|c|}
\hline \multirow[b]{2}{*}{$\begin{array}{l}\text { Fatores relacionados } \\
\text { ao tempo }\end{array}$} & \multicolumn{4}{|c|}{ Violência psicológica } & \multicolumn{4}{|c|}{ Violência física e psicológica } \\
\hline & $\%$ & OR & $\mathrm{IC}_{95 \%}(\mathrm{OR})$ & p-valor & $\%$ & OR & $\mathrm{IC}_{95 \%}(\mathrm{OR})$ & p-valor \\
\hline \multicolumn{9}{|l|}{ Duração do relacionamento } \\
\hline Menos de 1 mês & 28,7 & 1,00 & - & - & 23,3 & 1,00 & - & - \\
\hline Entre 1 mês e lano & 42,8 & 2,82 & $0,92-8,68$ & 0,010 & 36,0 & 1,34 & $0,49-3,72$ & 0,245 \\
\hline Mais de 1 ano & 28,5 & 5,22 & $2,07-13,12$ & 0,010 & 40,7 & 2,91 & $0,81-10,40$ & 0,245 \\
\hline \multicolumn{9}{|l|}{ Idade que ficou } \\
\hline 13 a 16 anos & 49,5 & 1,00 & - & - & 54,0 & 1,00 & - & - \\
\hline 8 a 12 anos & 50,5 & 1,88 & $0,86-4,14$ & 0,100 & 46,0 & 0,92 & $0,48-1,76$ & 0,765 \\
\hline \multicolumn{9}{|l|}{ Idade que namorou } \\
\hline 13 a 19 anos & 81,0 & 1,00 & - & - & 83,8 & 1,00 & - & - \\
\hline 8 a 12 anos & 19,0 & 1,49 & $0,31-7,11$ & 0,574 & 16,2 & 0,84 & $0,34-2,09$ & 0,673 \\
\hline \multicolumn{9}{|l|}{ Idade que transou } \\
\hline 15 a 18 anos & 73,6 & 1,00 & - & - & 71,1 & 1,00 & - & - \\
\hline 10 a 14 anos & 26,4 & 0,60 & $0,15-2,39$ & 0,417 & 28,9 & 1,09 & $0,45-2,66$ & 0,832 \\
\hline \multicolumn{9}{|l|}{$\begin{array}{l}\text { Perpetrou violência verbal } \\
\text { em relacion. anteriores }\end{array}$} \\
\hline Não & 72,3 & 1,00 & - & - & 47,2 & 1,00 & - & - \\
\hline Sim & 27,7 & 1,88 & $1,07-3,30$ & 0,031 & 52,8 & 4,65 & $2,10-10,28$ & 0,001 \\
\hline \multicolumn{9}{|l|}{$\begin{array}{l}\text { Perpetrou violência física } \\
\text { em relacion. anteriores }\end{array}$} \\
\hline Não & 95,1 & 1,00 & - & - & 85,0 & 1,00 & - & - \\
\hline $\operatorname{Sim}$ & 4,9 & 2,57 & $0,34-19,49$ & 0,304 & 15,0 & 9,45 & $1,63-54,71$ & 0,008 \\
\hline \multicolumn{9}{|l|}{$\begin{array}{l}\text { Perpetrou violência sexual } \\
\text { em relacion. anteriores }\end{array}$} \\
\hline Não & 98,4 & 1,00 & - & - & 97,9 & 1,00 & - & - \\
\hline Sim & 1,6 & * & * & 0,272 & 2,1 & 1,85 & $0,11-32,47$ & 0,633 \\
\hline
\end{tabular}

* Odds Ratio não calculado: casela igual a zero.

tes violentos no comportamento agressivo de adolescentes.

A duração do relacionamento também mostrou associação com violência psicológica, com aumento de chances de 5,81 em relacionamentos com duração maior que um ano. As pesquisas têm constatado que quanto mais envolvidos ${ }^{36} \mathrm{e}$ comprometidos $^{40}$ os namorados maior o risco de violência no namoro, o que está relacionado com o a duração deste.

Para a coocorrência de violência física e psicológica, a associação com a variável "ter sofrido violência física em relacionamentos anteriores” teve OR ${ }^{\text {ajustado }}$ de 12,55 , enquanto ter perpetrado violência verbal em relacionamentos anteriores teve OR ${ }^{\text {ajustado }}$ de 4,69 . Sears et al..$^{18}$ também investigaram a experiência passada de violência no namoro, como vítima ou perpetrador, como fator de risco para perpetração de violência no namoro atual de adolescentes e verificaram que ter sido vítima ou perpetrador de violência física ou psicológica em relacionamentos anteriores é fator de risco para a perpetração de violência física ou psicológica no namoro, tanto para homens como para mulheres.

Outros fatores associados com a coocorrência de violência física e psicológica foram sofrer violência física do pai $\left(\mathrm{OR}^{\text {ajustado }}\right.$ de 3,49$)$ e violência física entre irmãos ( $\left.\mathrm{OR}^{\text {ajustado }} \mathrm{de} 2,60\right)$. RiveraRivera et al. ${ }^{34}$, em um estudo com 7.960 adolescentes mexicanos, concluíram que o resultado mais importante foi o risco observado até 2 vezes maior de perpetração (e vitimização) de violência no namoro entre os adolescentes que sofreram violência familiar, principalmente se a violência intrafamiliar foi severa. Outros estudos corroboram esse resultado ${ }^{38,39,43}$. Méndez e Hernánde $z^{44}$ também observaram que a violência física perpetrada pelo pai é a mais determinante.

O estudo apresenta limitações com relação ao tamanho da amostra que foi calculada pelo parâmetro de prevalência. Outra limitação diz 
Tabela 5. Modelo final para os fatores associados à violência em adolescentes namorados. Análise multivariada. Recife, 2008.

\begin{tabular}{|c|c|c|c|c|}
\hline & \multicolumn{2}{|c|}{ Violência psicológica } & \multicolumn{2}{|c|}{ Violência física e psicológica } \\
\hline & $\begin{array}{l}\text { ORajustada } \\
{\left[\mathrm{IC}_{95 \%}(\mathrm{OR})\right]}\end{array}$ & p-valor & $\begin{array}{l}\text { ORajustada } \\
{\left[\mathrm{IC}_{95 \%}(\mathrm{OR})\right]}\end{array}$ & p-valor \\
\hline \multicolumn{5}{|l|}{ Fatores de processo } \\
\hline \multicolumn{5}{|l|}{$\begin{array}{l}\text { Sofre violência na } \\
\text { comunidade }\end{array}$} \\
\hline Não & 1,00 & - & & \\
\hline Sim & $3,99[1,61-9,87]$ & 0,007 & & \\
\hline \multicolumn{5}{|l|}{ Sofre violência física do pai } \\
\hline Não & & & 1,00 & - \\
\hline Sim & & & $3,49[1,23-9,90]$ & 0,024 \\
\hline \multicolumn{5}{|l|}{ Violência física entre irmãos } \\
\hline Não & & & 1,00 & - \\
\hline Sim & & & $2,60[1,01-6,71]$ & 0,048 \\
\hline \multirow{2}{*}{\multicolumn{5}{|c|}{$\begin{array}{l}\text { Sofreu violência física em } \\
\text { relacionamentos anteriores }\end{array}$}} \\
\hline & & & & \\
\hline \multicolumn{5}{|l|}{ Não } \\
\hline Sim & & & 1,00 & - \\
\hline Fatores relacionados ao tempo & & & $12,55[1,69-93,40]$ & 0,019 \\
\hline \multicolumn{5}{|l|}{ Duração do relacionamento } \\
\hline Menos de 1 mês & 1,00 & - & & \\
\hline Mais de 1 ano & $5,81[2,35-14,40]$ & 0,007 & & \\
\hline \multicolumn{5}{|l|}{$\begin{array}{l}\text { Perpetrou violência verbal em } \\
\text { relacionamentos anteriores }\end{array}$} \\
\hline Não & & & 1,00 & - \\
\hline Sim & & & $4,69[1,64-13,40]$ & 0,009 \\
\hline
\end{tabular}

respeito ao desenho transversal do estudo que não permite fazer inferências de causalidade.

Entretanto, a alta prevalência de violência no namoro observada no Recife se insere em um contexto de extrema violência urbana que a cidade apresenta ${ }^{45}$ e, dessa forma, esses resultados podem colaborar com a reflexão do tema em outras cidades brasileiras que partilham das mesmas características socioeconômicas e culturais. 


\section{Referências}

1. Follingstad DR, Bradley RG, Laughlin JE, Burke L. Risk Factors and Correlates of Dating Violence: The Relevance of Examining Frequency and Severity Levels in a College Sample. Violence Vict 1999; 14(4):365-380.

2. O’Leary KD, Barling J, Arias I, Rosenbaum A. Prevalence and stability of physical aggression between spouses: a longitudinal analysis. J Consult Clin Psychol 1989; 57(2):263-268.

3. Levy B. Abusive teen dating relationship: An emerging issue for the 90s. Response to the Victimization of Women and Children 1990; 13(1):59.

4. Ashley OS, Foshee VA. Adolescent help-seeking for dating violence: prevalence, sociodemographic correlates, and sources of help. J Adolesc Health 2005; 36(1):25-31.

5. Black BM, Tolman RM, Callahan M, Saunders DG, Weisz AN. When will adolescents tell someone about dating violence victimization? Violence Against Women $2008 ; 14(7): 741-758$

6. Howard DE, Wang MQ. Psychosocial factors associated with adolescent boys' reports of dating violence. Adolescence 2003; 38(151):519-533.

7. Foshee V. Gender differences in adolescent datinhg abuse prevalence, types and injuries. Health Educ Res 1996; 11(3):275-286.

8. Wolfe DA, Scott K, Reitzel-Jaffe D, Grasley C, Straatman AL, Wekerle C. Development and validation of the conuict in adolescent dating relationship inventory. Psychol Assess 2001; 13(2):277-293.

9. Kinsfogel KM, Grych JH. Interparental conûict and adolescent dating relationships: integrating cognitive, emotional, and peer inûuences. J Fam Psychol 2004; 18(3):505-515.

10. O'Leary KD, Slep AMS, Avery-Leaf S, Cascardi M. Gender differences in dating aggression among multiethnic high school students. J Adolesc Health 2008; 42(5):473-479.

11. Cyr M, Mcduff P, Wright J. Prevalence and predictors of dating violence among adolescent female victims of child sexual abuse. J Interpers Violence 2006; 21(8):1000-1016.

12. Schiff M, Zeira A. Dating violence and sexual risk behaviors in a sample of at-risk Israeli youth. Child Abuse Negl 2005; 29(11):1249-1263.

13. Sherer P, Sherer M. Exploring reciprocity in dating violence among Jewish and Arab youths in Israel. Int J Intercult Relat 2008; 32(1):17-33.

14. O'Keefe M. Teen dating violence: a review of risk factors and prevention efforts. Harrisburg, PA: VAWnet, a project of the National Resource Center on Domestic Violence/Pennsylvania Coalition Against Domestic Violence. [Internet] 2005 Apr. [acessado 2009 out 04]. [about 12 p]. Available from: http:// www.vawnet.org/.

15. Straus MA. Prevalence of violence against dating partners by male and female university student worldwide. Violence Against Women 2004; 10(7):790811

16. Jackson SM. Issues in the dating violence research: a review of the literature. Aggress Violent Behav 1999; $4(2): 233-247$.
17. Sabina C, Straus MA. Polyvictimization by Dating Partners and Mental Health Among U.S. College Students. Violence Vict 2008; 23(6):667-682.

18. Sears HA, Byers ES, Price EL. The co-occurrence of adolescent boys' and girls' use of psychologically, physically, and sexually abusive behaviours in their dating relationships. J Adolesc 2007; 30(3):487-504.

19. Cano A, Avery-Leaf S, Cascardi M, O’Leary KD. Dating violence in two high school samples: discriminating variables. J Prim Prev 1998; 18(4):431-

20. 446

O'Leary KD, Slep AMS. A dyadic longitudinal model of adolescent dating aggression. J Clin Child Ado-

21. lesc Psychol 2003; 32(3):314-327.

Hickman LJ, Jaycox LH, Aronoff J. Dating violence among adolescents: prevalence, gender distribution, and preventing program effectiness. Trauma

22. Violence Abuse 2004; 5(2):123-42.

Foshee VA, Linder F, MacDougall JE, Bangdiwala S. Gender Differences in the Longitudinal Predic tors of Adolescent Dating Violence. Prev Med 2001;

23. 32(2):128-141.

Aldright T. Prevalência e cronicidade da violência física no namoro entre jovens universitários do Estado de São Paulo - Brasil. Psicologia Teoria e Prática 2004; 6(1):105-120

24. Minayo MCS, Assis SG, Njaine K, organizadores. Amor e violência: um paradoxo das relações de namoro e do 'ficar' entre jovens brasileiros. Rio de Janeiro: Editora Fiocruz; 2011

25. Brasil. Ministério da Saúde (MS). Conselho Nacional de Saúde. Resolução nº 196 de 10 de outubro de 1996. Diretrizes e Normas Regulamentadoras de Pesquisas Envolvendo Seres Humanos. Diário Oficial da União 1996; out 16.

26. Kirkwood BR. Essentials of Medical Statistics. Oxford: Blackwell Science; 1997.

27. Szklo R, Nieto FJ. Epidemiology, beyond the basics. Sudbury, MA: Jones \& Bartlett Publishers; 2000.

28. Bronfenbrenner U. The bioecological theory of human development. In: Bronfenbrenner U. Making humans beings human: bioecological perspectives on human development. Thousand Oaks: Sage Publications; 2005

29. Kahn T, Bermergui C, Yamada E, Cardoso FC, Fernandes F, Zacchi JM, et al. O dia-a-dia nas escola (violências auto-assumidas). São Paulo: Instituto Latino-Americano das Nações Unidas para a Prevenção do Delito e Tratamento do Delinqüente, Instituto Sou da Paz; 1999.

30. Straus MA. Measuring intrafamily conflict and violence: the conflict tactics (CT) scales. J Marriage Fam 1979; 41(1):75-88.

31. Avanci JQ, Assis SG, Santos NC, Oliveira RVC. Adaptação transcultural de escala de auto-estima para adolescentes. Psicol Reflex Crít 2007; 20(3):397-405.

32. Associação Brasileira de Empresas de Pesquisa. Critério de Classificação Econômica Brasil. [Internet] 2010. [acessado 2010 abr 21] Disponível em: http:/ /www.abep.org/novo/CMS/Utils/FileGenerate. $\operatorname{ashx}$ ? id $=46$ 
33. Amar AF. Dating violence: comparing victims who are also perpetrators with victims who are not. $J$ Forensic Nurs 2007; 3(1):35-41.

34. Rivera-Rivera L, Allen-Leigh B, Rodríguez-Ortega G, Chávez-Ayala R, Lazcano-Ponce E. Prevalence and correlates of adolescent dating violence: Baseline study of a cohort of 7960 male and female Mexican public school students. Prev Med 2007; 44(6):477-484.

35. Banyard VL, Cross C, Modecki KL. Interpersonal Violence in Adolescence: Ecological Correlates of Self-Reported Perpetration. J Interpers Violence 2006; 21(10):13-14

36. O'Keefe M. Predictors of dating violence among high school students. J Interpers Violence 1997; 12(4):546-568.

37. Swart L, Stevens MSG, Ricardo I. Violence in adolescents' romantic relationships: findings from a survey amongst school-going youth in a South African community. J Adolesc 2002; 25(4):385-395.

38. Foshee VA, Bauman KE, Linder GF. Family violence and the preparation of adolescent dating violence: Examining social learning and social control processes. J Marriage Fam 1999; 61(2):331-342.

39. Foshee VA, Karriker-Jaffe KJ, Reyes HLM, Ennett ST, Suchindran C, Bauman KE, Benefield TS. What Accounts for Demographic Differences in Trajectories of Adolescent Dating Violence? An Examination of Intrapersonal and Contextual Mediators. J Adolesc Health 2008; 42(6):596-604.

40. Burke P, Stets J, Pirog-Good M. Gender identity, self-esteem, and physical and sexual abuse in dating relationships. Soc Psychol Q 1988; 51(3):272285.

41. Malik S, Sorenson SB, Aneshensel CS. Community and dating violence among adolescents: Perpetration and victimization. J Adolesc Health 1997; 21(5):291-302.

42. Gabarino J. Why adolescents are violent? Ciênc. saúde coletiva 2009; 14(2):533-538.

43. Rosen KH, Bartle-Haring S, Stith SM. Using bowen theory to enhance understanding of the intergenerational transmission of dating violence. J Fam Issues $2001 ; 22(1): 124-140$.

44. Méndez RG, Hernández JDS. La violencia en parejas jóvenes. Psicothema 2001; 13(1):127-131.

45. Waiselfisz JJ. Mapa da Violência 2011: os jovens do Brasil. [Internet] 2011. [acessado 2011 ago 10]. Disponível em: http://www.sangari.com/midias/pdfs/ MapaViolencia2011.pdf

Artigo apresentado em 05/05/2011

Aprovado em 28/08/2011

Versão final apresentada em 28/10/2011 